\title{
External Lightning Protection System for Wind Turbine Blades - Preliminary Aerodynamic Results
}

\author{
A. S. Ayub ${ }^{1}$, W. H. Siew ${ }^{2}$, S. J. MacGregor ${ }^{3}$ \\ Department of Electronic \& Electrical Engineering, \\ University of Strathclyde, \\ Glasgow, Scotland, UK \\ ahmad.ayub@strath.ac.uk ${ }^{1}$; wh.siew@strath.ac.uk ${ }^{2}$; scott.macgregor@strath.ac.uk ${ }^{3}$
}

\begin{abstract}
In general, there are three components making up a lightning protection system for wind turbines. These are the receptors, the down conductor and the grounding grid. Receptors and down conductors are usually found in the more recent wind turbine blades and where the down conductors are normally installed on the internal side of the blade. Consequently, the blades are vulnerable to damage and burn resulting from lightning strikes. The authors believe that a system with an external down conductor is likely to reduce the risk of damage when compared to the system having an internal down conductor. One could envisage an external down conductor would look similar to the one installed on a building or an aircraft. However, external down conductors may compromise the aerodynamic performance of the turbine blades. This paper reports the effect of external down conductors on the pressure coefficient distribution around the turbine blade. The blade profile (aerofoil) used is according to NACA 4418. Numerical simulations, using computational fluid dynamics (CFD), were conducted on an aerofoil without and with external down conductors of $1 \mathrm{~mm}$ thickness. The $\mathrm{k}-\varepsilon$ turbulence model that is incorporated in COMSOL Multiphysics (CFD Module) was used for the simulation and the wind speed and angle of attack used was $5 \mathrm{~m} / \mathrm{s}$ and $5^{\circ}$ respectively. The preliminary results show that the degradation on aerodynamic properties may not be too significant and these indicate that external down conductor arrangement could be considered.
\end{abstract}

Keywords- wind turbine blades; lightning protection; aerodynamic property; $k-\varepsilon$ turbulence model; computational fluid dynamics

\section{INTRODUCTION}

Essentially, there are three elements in a lightning protection system (LPS) for wind turbine. These are lightning receptors (also called air termination points), lightning down conductors and grounding arrangement in the soil of each wind turbine. The method of installation is adapted from practices in other industries (e.g. buildings and aircraft) [1, 2]. The main difference is the bonding network arrangement which depends on the geometry of the structure itself.

Furthermore, the development of lightning protection systems for wind turbines has increased in importance in the last 10 years and which culminated in the production of an International Standard in 2010 [1]. This document provides guidelines on how to integrate the different parts of a lightning protection system on a wind turbine to obtain the highest reliability.

The lightning receptors and down conductors associated with wind turbine blades may be installed, as suggested by the standard, on the internal or external side of the blade's surfaces [1]. Despite the choice available, manufacturers have opted to install the down-conductors on the internal side of a blade surface due to the perceived degradation of the aerodynamic properties of the blades' surfaces [1, 3]. Typically, the system that is often implemented by the wind turbine blades manufacturers is the placement of the lightning receptors on the surface of wind turbine blades but the lightning down conductor is placed internally in the blades [1, $3]$, as depicted in Figure 1. However, by having an internal down conductor, other problems occur (e.g. blade disintegration, burn) due to the impact of lightning strikes [1].

Therefore, in the attempt to reduce the likelihood of this particular event, a group of researchers from the University of Strathclyde, Scotland [4-6] has questioned whether the installation of receptor and down conductor on the external surface of the blade is preferable.

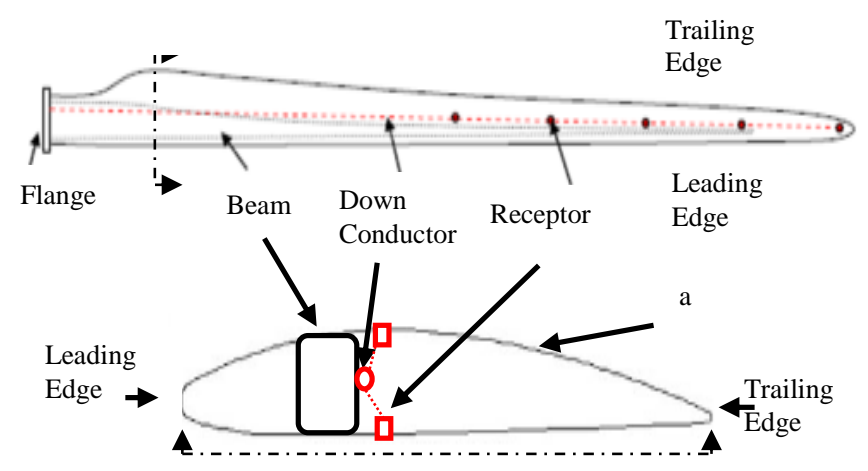

Figure 1 Typical Lightning Receptors and Internal Down Conductor System Installation $-2 \mathrm{D}$ view (i.e. a, a') from blade's root, adapted from $[1,3]$

An external lightning protection system on the blade's surfaces is likely to compromise the aerodynamic properties of the blade but the system would be more effective in providing lightning attachment points. The installation of such a system on the external surface of the blade is likely to affect the 
smooth (i.e. streamline) wind flow due to the protrusion of the down-conductor above the surface of the blade. A disturbed (i.e. turbulent) wind flow would also compromise the overall performance of the turbine blade itself (i.e. aerodynamic properties) [1].

Previous experimental and numerical findings by other researchers addressed surface roughness due to ice accretion and dust accumulation on aerofoil surfaces; particularly on the leading edge where the roughness was just below $1 \mathrm{~mm}[7,8]$. On the other hand, the Standards [1] has recommended that the typical cross section for down conductor is $50 \mathrm{~mm}^{2}$ when considering lightning protection system. Generally, this is achieved practically (i.e. down conductor for building) by having a rectangular cross-section and where the thickness is greater than $1 \mathrm{~mm}$ (or equal). Consequently, previous findings are not completely helpful in assessing the effect of the higher protrusions in various positions on the aerofoil surfaces. Hence, the uncertainty is addressed by the authors and this paper discussed the progress of the investigation on aerodynamic studies (i.e. pressure coefficient distribution) when considering external lightning protection systems (LPS) for aerofoils.

Modelling of fluid (i.e. wind) flow field around wind turbine blades in 3 dimensions is a challenging task. Furthermore, the available turbulence models have yet to demonstrate acceptable level of stability that correctly predicts the results for turbulent flow [9]. Therefore, the wind flow in this study is considered to be turbulent (due to high Reynolds Number - order of $10^{6}$ ), incompressible (i.e. constant flow density) and only for two dimensional (2D) geometries (i.e. aerofoil). The incompressible flow refers to the flow density as being constant throughout the aerofoil where the large pressure changes and high wind speed (exceeds Mach number 0.3) are insignificant, thus they can be ignored. Furthermore, although the considered simulation uses 2D for its geometry, the results produced are still valid due to similar airflow characteristic (determined by dimensionless Reynolds number) with three dimensional (3D) geometries [10-15]. In other words, the investigation based on a $2 \mathrm{D}$ model is still valid as long as the Reynolds number remained similar to that of the $3 \mathrm{D}$ geometry.

In the following sections, this paper will provide a concise review of wind flow around an aerofoil. The paper then discusses the numerical modelling methodology (i.e. turbulence modelling) where simulations for clean aerofoil surfaces were first studied. Then, the protruded aerofoil surfaces were investigated followed by analyses and discussions of the results. Finally, conclusions were drawn and future work proposed.

\section{REVIEW OF AERODYNAMIC BEHAVIOUR}

In this section, the fundamental description concerning the aerodynamic properties of an aerofoil is concisely presented so as to provide an overview of the subject under investigation. This includes the introduction of aerofoil's terminology and the concept of wind flow behaviour around aerofoil surfaces.
Further information on the above-mentioned sub-topics is widely available in textbooks [10, 11, 13-15].

\section{A. Aerofoil Geometry and its Terminology}

A cross section of aerofoil geometry is drawn in two dimensions (2D) and its terms are labelled as illustrated in Figure 2. There are 2 components associated with an aerofoil in terms of aerodynamic properties, which are lift (L) coefficient and drag (D) coefficient. Lift is the component that is perpendicular to the oncoming flow direction whilst drag is the component that is in parallel with the oncoming flow. Both of which are created from the wall shear stresses at each of aerofoil profile points (at lower and upper surfaces) where the forces are called lift and drag forces. The performance of an aerofoil profile is determined by ratio between generated lift and drag when an aerofoil moves through the air and it is called lift to drag (L/D) ratio. The L/D ratio is one of the important parameters in an aerofoil design such as glider, aircraft and wind turbine blade [10, 11, 14, 15].

\section{B. Wind Flow Around an Aerofoil Surface - Brief Concept}

In general, the air flow around an aerofoil surface of wind turbine blades is similar to an aircraft wing. As airflow meets the leading edge of the aerofoil, as illustrated in Figure 2, it separates. Part of it goes over (i.e. upper surface) and the rest goes under (i.e. lower surface) the aerofoil respectively.

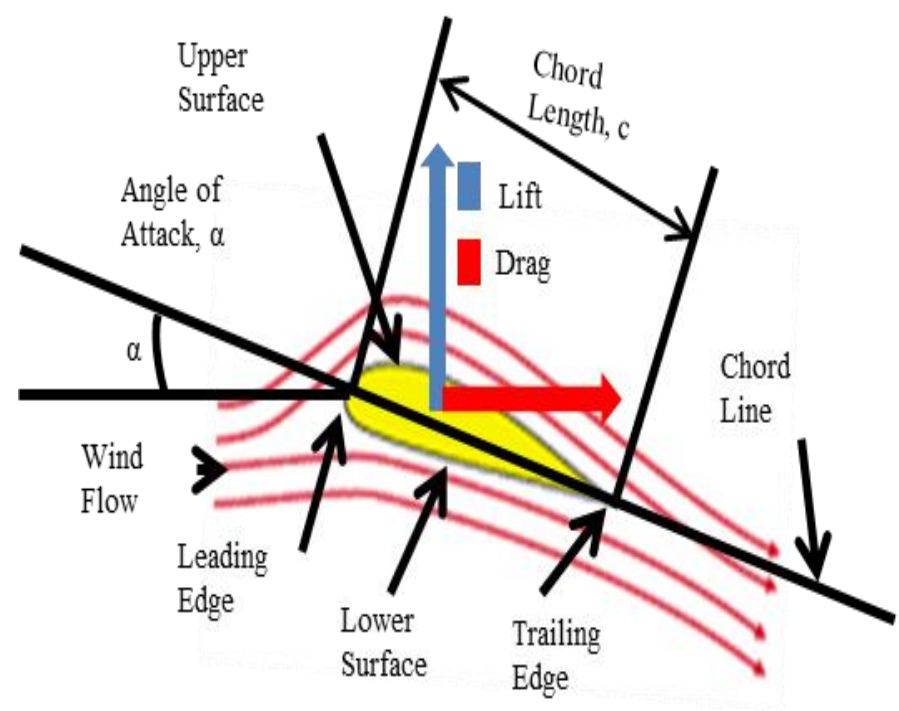

Figure 2 Cross section of aerofoil geometry (2D) and its terms, adapted from $[10,11]$

Since the upper surface is more curved than the lower surface (i.e. cambered aerofoil), it creates lower pressure on the upper surface (also called suction side) while higher pressure is created on the lower surface (also called pressure side), thus, generating lift as wind passes it. Furthermore, the lift force can be dramatically increased by changing its angle (i.e. angle of attack) to the wind. However, the aerofoil stalls at very large angles of attack as the lift force gradually decreases. This behaviour is due to the retarding force called 
drag in which it also increases with angles of attack. Figure 3 illustrates the behaviour of wind flow around an aerofoil surface with respect to different angles of attack.

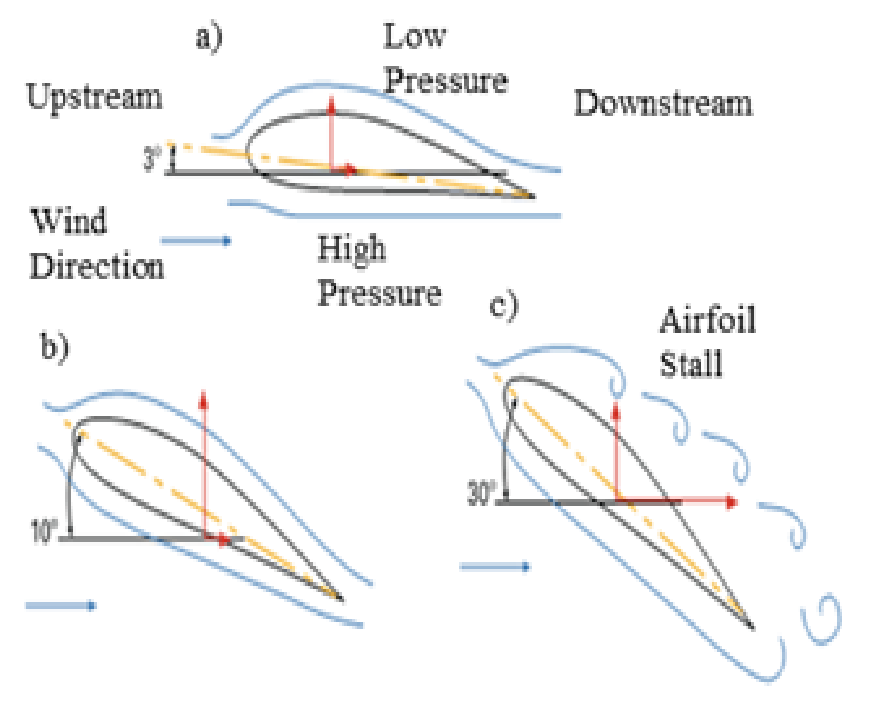

Figure 3 The behaviour of wind flow around an aerofoil surface with respect to different angles of attack; a) low, b) medium and c) high, adapted from [10, 11]

Owing to that, lift and drag forces are significantly influenced by the pressures created on either at the lower or upper surfaces of an aerofoil. In light of pressures created around an aerofoil, it can be quantified by the dimensionless pressure coefficient, $C_{p}[10,11,14]$. As written in (1), pressure coefficient describes relative pressure throughout the wind flow field around an aerofoil particularly in the flow adjacent to the aerofoil surfaces itself $[10,11]$.

$$
C_{p}=\frac{p-p_{\infty}}{\frac{1}{2} \rho_{\infty} V_{\infty}^{2}}
$$

Where $p$ is the pressure at the point at which pressure coefficient is being calculated, $p_{\infty}$ is the pressure in the free stream wind flow, $\rho_{\infty}$ is the fluid density (in this case is air which is $1.2 \mathrm{~kg} / \mathrm{m}^{3}$ ) and $V_{\infty}$ is the velocity of the wind.

In aerodynamics performance analysis, this pressure coefficient value is normally plotted in the form of pressure coefficient distribution (see Figure 5-7) starting from leading edge to trailing edge of an aerofoil. Section IV describes in more details how such a plot is used.

\section{NUMERICAL MODELLING}

In this section, the numerical technique utilised in this investigation are concisely explained. Hence, further explanation on the subject is widely available in textbooks [9, 16]. Furthermore, the modelling technique of the investigation is also presented.

\section{A. Numerical Technique}

\section{A.1. Governing Equations}

A standard $\mathrm{k}-\varepsilon$ turbulence model is utilised in COMSOL Multiphysics (CFD Module) [16] as it is one of the most used turbulence models for industrial applications. This model introduces two dependant variables equations (i.e. Turbulent Kinetic Energy, k and Dissipation Rate of Turbulence Energy, $\varepsilon$ ) which are written as given in (2) and (3) respectively.

Turbulent Kinetic Energy

$$
\frac{\partial k}{\partial t}+U_{j} \frac{\partial k}{\partial x_{j}}=\tau_{i j} \frac{\partial U_{i}}{\partial x_{j}}-\varepsilon+\frac{\partial}{\partial x_{j}}\left[\left(v+v_{T} / \sigma_{k}\right) \frac{\partial k}{\partial x_{j}}\right]
$$

Dissipation Rate of Turbulence Energy

$$
\begin{aligned}
& \frac{\partial \varepsilon}{\partial t}+U_{j} \frac{\partial \varepsilon}{\partial x_{j}}=C_{\varepsilon 1} \frac{\varepsilon}{k} \tau_{i j} \frac{\partial U_{i}}{\partial x_{j}}-C_{\varepsilon 2} \frac{\varepsilon^{2}}{k} \\
& +\frac{\partial}{\partial x_{j}}\left[\left(v+v_{T} / \sigma_{\varepsilon}\right) \frac{\partial \varepsilon}{\partial x_{j}}\right]
\end{aligned}
$$

where its closure coefficients are: $\mathrm{C}_{\varepsilon 1}=1.44, \mathrm{C}_{\varepsilon 2}=1.92$, $\mathrm{C}_{\mu}=0.09, \sigma_{\mathrm{k}}=1.0$ and $\sigma_{\varepsilon}=1.3$,

\section{B. Modelling Technique-Model Configuration and Dimension}

For this study, the model is simplified with the following simplifications: the flow is two dimensional, incompressible and turbulent (due to high Reynolds number - order of $10^{6}$ ). A NACA 4418 aerofoil profile was selected [10] for all simulation cases (i.e. with and without protrusions) and stationary-state simulations were performed. The whole computation zone consists of air domain with a dimension of $100 \mathrm{~m}$ height $\times 150 \mathrm{~m}$ width and the selected aerofoil (with 5 $\mathrm{m}$ of chord length) is placed at $35 \mathrm{~m}$ and $115 \mathrm{~m}$ from the inlet and outlet respectively, as depicted in Figure 4. In addition, the aerofoil is placed in the middle of the air domain (i.e. $50 \mathrm{~m}$ in between top and bottom walls). The boundaries were set to avoid perturbation coming from the domain limits and to allow the air flow to be fully extended. Furthermore, the wind speed and angle of attack used in simulations are $5 \mathrm{~m} / \mathrm{s}$ (i.e. cut-in wind speed for most modern wind turbine) and $5^{\circ}$ (i.e. highest L/D ratio for NACA 4418) respectively.

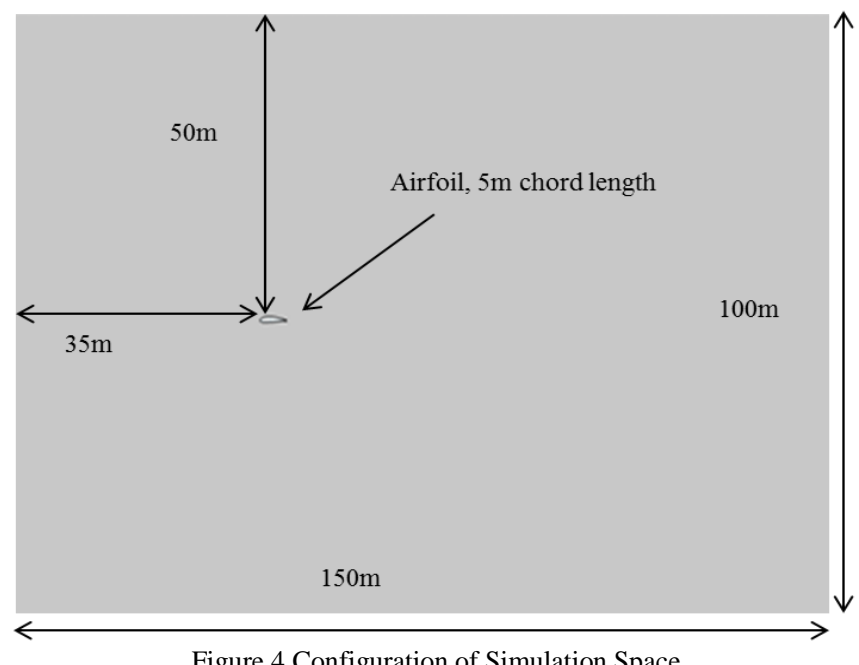

Figure 4 Configuration of Simulation Space 
In general, meshing for the simulations was configured using free triangular meshes with fine meshes in the vicinity of aerofoil surfaces and coarser meshes towards the outer boundary of the air domain. The model was simulated for two cases which are: without protrusion (i.e. no external down conductors) and with protrusions (i.e. comprising external down conductors).

\section{B.1. Model without protrusions - Clean Aerofoil Surfaces}

Further to the model configuration, the clean aerofoil was simulated. The results of aerodynamic properties were used for comparison with model with protrusions.

\section{B.2. Model with protrusions - Protruded Aerofoil Surfaces}

The protrusion (i.e. down conductor) dimension is configured to comply with typical cross section (i.e. $50 \mathrm{~mm}^{2}$ ) as recommended by IEC 61400-24 [1]. Hence, the down conductor has been configured with $1 \mathrm{~mm}$ height and $50 \mathrm{~mm}$ width (i.e. rectangular shape). The model considered for two scenarios where it allowed the authors to visualise the effect of protrusions location on the aerodynamic performance.

For the first scenario (i.e. single conductor), the protrusions were first placed at $1 \mathrm{~m}$ from the leading edge on upper and lower aerofoil surfaces. With the same protrusion height, the simulation was then continued with other scenarios (i.e. multiple conductors) where the protrusions were placed at intervals of $1 \mathrm{~m}$ between each other on upper and lower aerofoil surfaces. In all cases, the protrusions were perpendicular to the chord length.

\section{SIMULATION RESULTS AND DISCUSSIONS}

Apart from lift and drag coefficients, pressure coefficient is also an important parameter when analysing the aerodynamics performance of an aerofoil especially when considering surface roughness (i.e. protrusion) sensitivity studies (or designing one) [7, 8, 12, 17]. Furthermore, the pressure coefficient distribution also provides useful information when considering aerofoil profiles modification (or alteration) to achieve a specific objective. Normally, in aerofoil profiles modification, different aerofoil profiles are being compared (in terms of pressure coefficient distribution) and altered (or perhaps fine-tuned) to achieve better lift to drag ratio $[7,8,12,18,19]$ (e.g. glider, wind turbine blade).

With the idea of surface roughness sensitivity study and aerofoil profile modification, the results of pressure coefficient distribution as obtained in this paper will be used to suggest possible locations (on aerofoil surfaces) for the return conductors of an external lightning protection system.

Note that the pressure coefficient (on the y-axis in Figure $5-7$ ) is plotted "upside-down" with negative values higher on the plot. This is done so that the upper surface of an aerofoil corresponds to the upper curve in the pressure coefficient plot. Likewise, the lower surface of an aerofoil corresponds to the lower curve in the pressure coefficient plot. Plots of pressure coefficient distribution for each case are shown and discussed as follow where the aerofoil is at $5^{\circ}$ angle of attack with wind flow of $5 \mathrm{~m} / \mathrm{s}$.

\section{A. Model without Protrusions - Clean Aerofoil Surfaces}

Figure 5 shows that the pressure coefficient started from about 1.0 at the stagnation point (i.e. zero local wind velocity, thus highest pressure) near the leading edge due to incompressible flow behaviour. The minimal value of pressure coefficients are about -1.6 (i.e. lowest pressure) and 0.1 for upper and lower surfaces respectively. The plot curves then increased rapidly (i.e. pressure decreases) for both upper and lower surfaces and finally recovered to a small positive value (i.e. 0.2) of pressure coefficients near the trailing edge. These pressure coefficient values (i.e. negative and positive values) correspond to the wind flow behaviour (i.e. low pressure for upper surface and high pressure for lower surface) accordingly, as previously depicted in Figure 3. Furthermore, since the aerofoil is pitched at $5^{\circ}$ angle of attack (i.e. low angle of attack in Figure 3a), the likelihood of the aerofoil to stall is highly unlikely.

Overall, the pressure coefficients show reasonably smooth loci throughout the aerofoil surface.

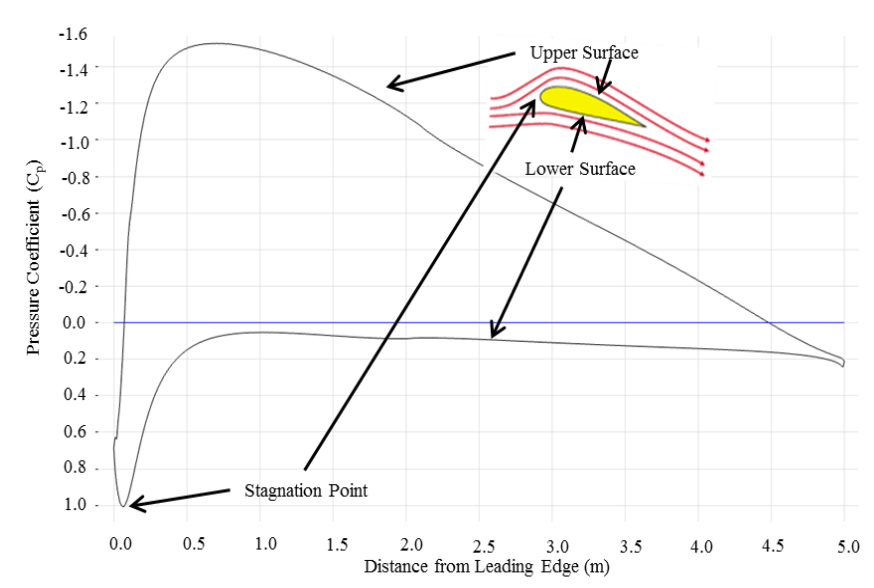

Figure 5 Pressure coefficient distributions of clean aerofoil surface (i.e. no conductor) at $5 \mathrm{~m} / \mathrm{s}$ of wind speed and at $5^{\circ}$ angle of attack- inset image of an aerofoil and wind direction are for easy reference.

\section{B. Model with Protrusions - Protruded Aerofoil Surfaces}

\section{B.1. Protrusions at 1 from the leading edge-Single Conductor}

The pressure coefficient distribution for a single conductor located at $1 \mathrm{~m}$ from the leading edge for upper and lower aerofoil surface is as shown in Figure 6. It can be seen that the loci of pressure coefficient distributions contain discontinuities (i.e. glitches) due to the presence of protrusions at both upper and lower surfaces. Furthermore, the wind flow is very much separated at the front and back ends of the protrusions (i.e. conductors), hence the discontinuities.

Furthermore, the lowest pressure coefficient values for both surfaces are respectively at -2.6 for upper and -0.4 for lower as it is at the front end of conductors (i.e. protrusions) shape itself where the wind flow are first separated. As wind flows over the conductor, it shows that the pressure coefficient values for both surfaces are less severe as the flow was likely 
to recover momentarily on the conductor's surface (i.e. width of $5 \mathrm{~cm})$. Then, as wind flows to the back end of the conductor and finally reattached to the aerofoil surfaces, it appeared to have a smaller glitch when compared to the earlier one with the magnitude of pressure coefficient values for both surfaces at -1.8 for upper and -0.1 for lower respectively. The pressure coefficients are swiftly restored to the smooth loci towards the trailing edge where it finally recovered to the same positive coefficient value (i.e. 0.2) as of the clean (i.e. no conductor) case.

Furthermore, the conductors (i.e. single conductor case) located at the upper and lower surfaces have considerably reduced the aerodynamic performances of the aerofoil, as tabulated in TABLE 1.

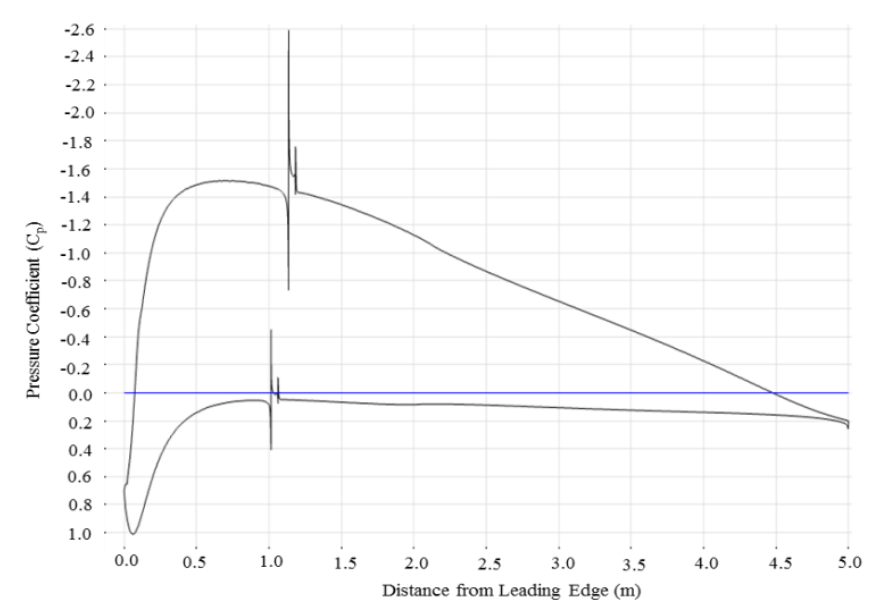

Figure 6 Pressure coefficient distributions of $1 \mathrm{~mm}$ protrusions at $1 \mathrm{~m}$ from leading edge for upper and lower aerofoil surfaces (i.e. single conductor) for $5 \mathrm{~m} / \mathrm{s}$ of wind speed and at $5^{\circ}$ angle of attack

\section{B.2. Protrusion perpendicular to the chord length-} Multiple Conductors

The pressure coefficient distributions for multiple conductors' located perpendicular to the chord length are as plotted in Figure 7. It is found that the glitch occurred at $1 \mathrm{~m}$ interval where the conductors are placed. However, its pressure coefficient values are varied depending on the location of the conductors itself. Furthermore, the pressure coefficient value at stagnation point is found to be similar (i.e. 1.0) to the previous cases (i.e. clean and single conductors' case).

As the pressure coefficient values move from the stagnation point to the trailing edge, it is found that there is glitch occurred close to the stagnation point. This is understandably due to the presence of conductor placed at the leading edge. However, it only occurred on the upper curve of the plot which corresponds to upper surface of the aerofoil. This is due to the aerofoil being pitched (similar to the previous cases) at an angle of $5^{\circ}$ to the wind flow. Thus, the pressure distribution at stagnation point is not affected by the conductor being placed at the leading edge.
Apparently, despite the fact that there is a conductor placed at the trailing edge, the pressure coefficient distribution at the trailing edge is found to be similar to the previous cases where it recovers to the same positive coefficient value (i.e. 0.2 ). It can be seen that the pressure coefficient values of conductors located at $3 \mathrm{~m}$ and $4 \mathrm{~m}$ on the suction side (i.e. upper surface) are relatively small when compared to conductors placed at $1 \mathrm{~m}$ and $2 \mathrm{~m}$ from leading edge. The pressure coefficient values for conductors placed on the surface of pressure side (i.e. lower surface) are also small and its magnitudes are about similar from one to another except the conductor located at $1 \mathrm{~m}$ from leading edge where it resembles similar pressure coefficient value to the single conductors' case. Thus, these findings indicate that the aerodynamic performance of the aerofoil is likely to be compromised by these locations (i.e. $1 \mathrm{~m}$ and $2 \mathrm{~m}$ for upper and $1 \mathrm{~m}$ for lower surface). With that in mind, these locations may not be viable (with respect to aerodynamics performance) for conductor's installation.

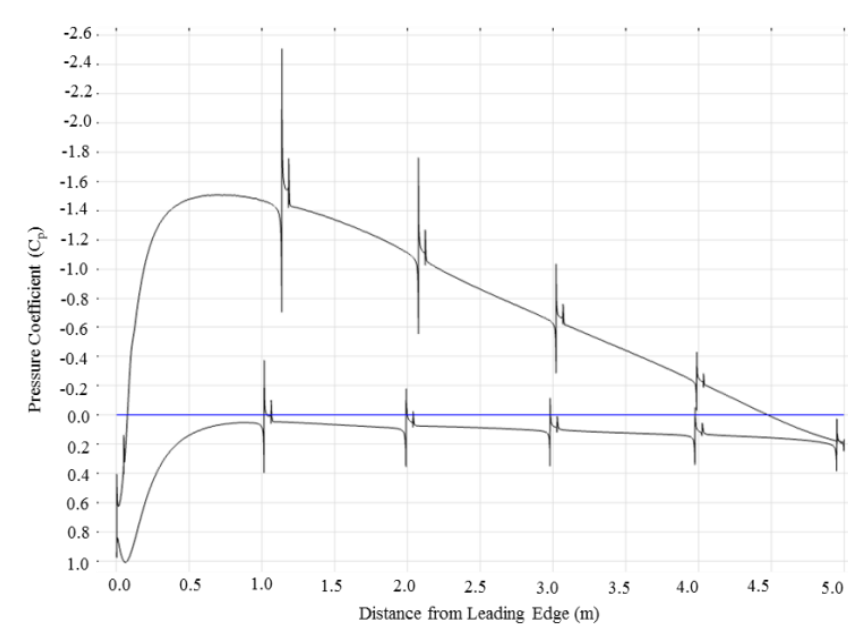

Figure 7 Pressure coefficient distributions of $1 \mathrm{~mm}$ protrusions placed perpendicular to the chord length (i.e. mulitple conductor at $1 \mathrm{~m}$ interval) for $5 \mathrm{~m} / \mathrm{s}$ of wind speed and at $5^{\circ}$ angle of attack.

Ignoring the glitches, the loci of the pressure coefficient distribution are similar when comparing results with and without protrusions. Thus, this suggests that the wind flows are swiftly recovered after each interruption by the conductors.

Furthermore, the performance of lift, drag and lift to drag ratio has, indeed, been greatly affected by these conductors, as tabulated in TABLE 1 .

TABLE 1 TABUlated DATA OF AERODYNAMics PROPERTIES FOR ClEAN, SinGLE AND MulTIPLE CONDUCTORS [6]

\begin{tabular}{|c|c|c|}
\hline L, Clean & L, Single & L, Multiple \\
\hline 0.962860 & 0.930474 & 0.942036 \\
\hline D, Clean & D, Single & D, Multiple \\
\hline 0.006809 & 0.008643 & 0.009335 \\
\hline L/D, Clean & L/D, Single & L/D, Multiple \\
\hline 141.4098 & 107.6563 & 100.9144 \\
\hline
\end{tabular}


In TABLE 1, the simulation results of lift to drag ratio for single and multiple conductors show a reduction in aerodynamic performance of $24 \%$ and $29 \%$ respectively when compared to the clean (i.e. no conductor) case.

\section{CONCLUSIONS AND FUTURE WORK}

Study on the aerodynamic properties of external lightning protection systems for wind turbine blades is presented. The concise review of aerodynamic properties is briefly discussed with respect to aerofoil geometry and its terminology and the wind flow behaviour around aerofoil surfaces. The numerical technique and modelling configurations are also discussed where $\mathrm{k}-\varepsilon$ turbulence model is used for all simulations using COMSOL Multiphysics (i.e. CFD Module).

An aerofoil based on NACA 4418 was used to carry out CFD study with (i.e. single and multiple conductors) and without (i.e. no conductors) protrusions on aerofoil surfaces where the wind speed used is $5 \mathrm{~m} / \mathrm{s}$ and angle of attack was $5^{\circ}$. The conductor used in all simulations was rectangular in cross-section having a height of $1 \mathrm{~mm}$ and $50 \mathrm{~mm}$ width and it is IEC 61400 Standards compliant.

The results of pressure coefficient distributions for all cases were compared and it is found that the effect on pressure coefficient distributions appeared to be local to where the conductors are placed. Therefore, in general, it can be concluded that the wind flows are swiftly recovered after each conductor.

Furthermore, with respect to aerodynamic performance, there are several locations on the aerofoil surfaces that could be considered to be viable for external conductor installation. Moreover, it is found that the multiple conductors case shows larger reduction (in terms of lift to drag ratio) in comparison to clean (i.e. no conductor) and single conductors cases. Thus, it can be concluded that single conductor arrangement is rather preferable due to smaller reduction in its lift to drag ratio.

Although single conductor arrangement is preferred based on this work, it may not be sufficient to provide adequate protection against lightning strikes onto wind turbine blades. Therefore, in the attempt to provide better lightning protection for wind turbine blade, an immediate extension of this work is to extend the study to different intervals between protrusions (i.e. conductors). It would also be interesting to perform the same analysis for different protrusion (i.e. conductor) height. The results of these future works will be very helpful to determine the best conductor's location (and also its height) with minimum reduction of aerodynamics performance whilst safeguarding a wind turbine blade from disastrous lightning impact.

\section{REFERENCES}

[1] IEC 61400-24:2010, "IEC 61400-24 Wind Turbines - Part 24: Lightning Protection", International Electrotechnical Commission (IEC), June 2010.

[2] V. Cooray, "Lightning Protection", IET Power \& Energy Series 58, The Institution of Engineering \& Technology (IET), London, 2010.

[3] LM Wind Power, "Available: http://www.lmwindpower.com/RotorBlades/Products/Features/Add-Ons/Lightning-Protection", Accessed: 9th Jan 2014

[4] A. S. Ayub, W. H. Siew, and S. J. Macgregror, "Lightning Protection of Wind Turbine Blades - An Alternative Approach", 7th Asia-Pacific International Conference on Lightning (APL 2011), Chengdu, China, Nov, 2011.

[5] A. S. Ayub, W. H. Siew, and S. J. Macgregor, "Proposed External Lightning Protection System for Wind Turbine Blades - Aerodynamic Properties", 8th Asia-Pacific International Conference on Lightning (APL 2013), Seoul, Korea, June, 2013.

[6] A. S. Ayub, W. H. Siew, and S. J. Macgregor, "External Lightning Protection System for Wind Turbine Blades - A Preliminary Study", International Colloquium on Lightning and Power Systems-CIGRE SC C4 on System Technical Performance, Lyon, France, May, 2014.

[7] F. Villalpando, M. Reggio, and A. Ilinca, "Numerical Flow Simulation over Clean and Iced Wind Turbine Blades", 17th Annual Conference of the CFD Society of Canada, Canada, May, 2009.

[8] N. Ren and J. Ou, "Dust Effect on the Performance of Wind Turbine Airfoils", Journal of Electromagnetic and Application, Issue 1, 2009, $102-107$.

[9] D. C. Wilcox, "Turbulence Modelling for CFD", DCW Industries, California, US, 1998.

[10] I. R. Abbott and A. E. V. Doenhoff, "Theory of Wing Sections including summary of airfoil data", Dover Publication, New York, 1959.

[11] John D. Anderson Jr., "Fundamental of Aerodynamics", McGraw Hill, New York, 2011.

[12] R. P. J. O. M. v. Rooij and W. A. Timmer, "Roughness Sensitivity Considerations for Thick Rotor Blade Airfoils", Transactions of the ASME, Vol. 125, Nov, 2003,

[13] F. M. White, "Viscous Fluid Flow", $2^{\text {nd }}$ Edition, McGraw Hill, 1991.

[14] Martin O. L. Hansen, "Aerodynamics of Wind Turbines", 2nd Edition, Earthscan, London, UK, 2007.

[15] T. Burton, D. Sharpe, N. Jenkins, et. al., "Wind Energy Handbook", John Wiley \& Sons Ltd, England, 2001.

[16] COMSOL Multiphysics. Ltd, "COMSOL Multiphysics - User's Guide", Nov, 2012.

[17] W. A. Timmer and A. P. Schaffarczyk, "The Effect of Roughness at High Reynolds Numbers on the Performance of Aerofoil DU97-W300Mod", Wind Energy, Vol. 7, Issue 4, 2004, pp. 295-307.

[18] F. Villalpando, M. Reggio, and A. Ilinca, "Numerical Study of Flow Around Iced Wind Turbine Airfoil", Journal of Engineering Applications of Computational Fluid Mechanics, Vol. 6, No. 1, 2012, pp. 39-45.

[19] K. Yang, L. Zhang, and J. Z. Xu, "Simulation of Aerodynamic Performance Affected By Vortex Generators on Blunt Trailing Edge Airfoils", Science China-Technological Sciences, Vol. 53, No. 1, Jan, 2010, pp. 1-7. 